\title{
Gestión de Residuos Sólidos Urbanos y su Impacto Medioambiental
}

\author{
Jacqueline Bartra Gómez \\ jbartrag@ucv.edu.pe \\ Escuela de posgrado \\ Universidad Cesar Vallejo \\ ORCID: 0000-0002-2745-1587 \\ José Manuel Delgado Bardales \\ jmdelgadob@ucvvirtual.edu.pe \\ Escuela de posgrado \\ Universidad César Vallejo \\ Tarapoto - Perú \\ ORCID: 0000-0001-6575-2759 \\ Scopus autor ID: 24070333700 \\ Código Renacyt: P0050554
}

\section{RESUMEN}

Esta investigación buscó caracterizar la gestión de residuos sólidos urbanos y el impacto ambiental que estas producen. El tipo de investigación es no experimental básica y el diseño de estudio es una revisión sistemática; las revisiones sistemáticas se realizaron de investigaciones científicas, cuya unidad de análisis se realizó en base a estudios originales primarios, los mismos que representan una herramienta principal para sintetizar la información científica disponible, incrementar la validez de las conclusiones de estudios individuales, así como dirimir las áreas de incertidumbre donde sea de prioridad realizar investigación. Respecto a la gestión de residuos sólidos urbanos; en donde a pesar de los esfuerzos que realizan algunas municipalidades, de acuerdo a lo investigado, se logró identificar entre los principales problemas: la distancia de los vertederos, el déficit de aprovechamiento, la disposición final de los desechos no está bien ubicada y mucho menos reutilizada, es por esta razón que hoy más que nunca debemos involucrarnos desde distintos ámbitos y promover la recolección selectiva, y trabajar fehacientemente una educación ambiental que contribuya eficientemente con el cuidado del medioambiente.

Palabras clave: desecho, medio ambiente, administración local. 


\title{
Management of Urban Solid Waste and its Environmental Impact
}

\begin{abstract}
This research sought to characterize the management of urban solid waste and the environmental impact they produce. The type of research is basic non-experimental and the study design is a systematic review; The systematic reviews were carried out of scientific investigations, whose unit of analysis was carried out based on original primary studies, which represent a main tool to synthesize the available scientific information, increase the validity of the conclusions of individual studies, as well as resolve the areas of uncertainty where research is a priority. Regarding the management of solid urban waste; Where despite the efforts made by some municipalities, according to the investigation, it was possible to identify among the main problems: the distance from the landfills, the deficit of use, the final disposal of the waste is not well located, much less reused, it is for this reason that today more than ever we must get involved from different areas and promote selective collection, and reliably work on an environmental education that efficiently contributes to caring for the environment.
\end{abstract}

Keywords: waste, environment, local administration.

Artículo recibido: 03 nov. 2020 Aceptado para publicación: 07 dic. 2020 Correspondencia jbartrag@ucv.edu.pe Conflictos de Interés: Ninguna que declarar 


\section{INTRODUCCIÓN}

El manejo de los residuos sólidos urbanos es un problema que amerita pronta solución en todo el mundo, el crecimiento poblacional desordenado es un factor que ha empeorado esta situación. El informe del Banco Mundial (2018), indica que el crecimiento de los desechos en el mundo crecerá en un 70\% hacia el 2050, es por ello la urgencia de tomar medidas de emergencia para intentar frenar esta situación. Los países de primer mundo si bien en cierto representan solo el 16\% de la población, éstos generan 34\% de los desechos, Asia y el Pacífico generan el $23 \%$ y en África se prevé que se expanda al triple para el 2050. Asimismo, los municipios cumplen un rol muy importante respecto del manejo de residuos sólidos urbanos, puesto que son ellas las encargadas de que se brinde este servicio a toda la población. Lamentablemente las malas gestiones de los residuos sólidos urbanos vienen perjudicando la salud del mundo y constituye un agravante en el cambio climático que afecta todo el planeta.

Sameh Wahba, afirma: "La gestión inadecuada de los desechos está produciendo la contaminación de los océanos del mundo, obstruyendo los drenajes y causando inundaciones, transmitiendo enfermedades, aumentando las afecciones respiratorias por causa de la quema, perjudicando a los animales que consumen desperdicios, y afectando el desarrollo económico, por ejemplo, al perjudicar el turismo”. Banco Mundial, 2018.

Según el Ministerio del Ambiente (MINAM), en el Perú se genera 19,000 toneladas diarias de residuos, lo que representa tres veces la capacidad actual del Estadio Nacional. Del total de residuos sólidos generados en el territorio nacional, el 52\% va a los 34 rellenos sanitarios autorizados, mientras que el $48 \%$ se vierte en 1,585 botaderos identificados que son lugares que ponen en riesgo la calidad del ambiente y la salud de los ciudadanos.

Las municipalidades son las encargadas del servicio de recolección de residuos sólidos urbanos, estimado en alrededor del $91.21 \%$ de la población urbana de todos los distritos del país, lo que equivale a 1867 municipios a nivel nacional, los mismos que en su mayoría no cuentan con un sistema de gestión de residuos sólidos urbanos implementados con los procesos de minimización y segregación en la fuente, hasta la disposición final o el reaprovechamiento de los mismos. La Municipalidad Provincial de San Martín atiende a 14 distritos, entre ellos el más importante es la ciudad de Tarapoto; esta atención se ha 
venido complicando respecto al manejo de los residuos sólidos urbanos, debido al desordenado crecimiento que se ha producido en la ciudad, por causa de las numerosas invasiones que se han venido dando en los últimos años.

La gestión municipal hoy en día tiene un reto muy grande para poder dar la atención debida a toda la población, respecto del manejo de los residuos sólidos urbanos, que se producen diariamente, es por ello que se formuló el siguiente problema de investigación ¿cuáles son las características de la gestión de residuos sólidos urbanos y el impacto en el medio ambiente? Asimismo, el objetivo principal de la investigación fue caracterizar la gestión de Residuos Sólidos Urbanos y el impacto ambiental que estas producen.

\section{ESTRATEGIAS METODOLÓGICAS O MATERIALES Y MÉTODOS}

El tipo de investigación será de carácter no experimental básica; y se realizará una revisión sistemática de la información, las revisiones sistemáticas son investigaciones científicas en las que se analizan los estudios originales primarios, por lo que constituyen una herramienta útil para sintetizar la información científica disponible, mejorar la validez de las conclusiones de estudios individuales e identificar áreas que se consideren imprecisas y donde se requiera realizar una investigación.

La recolección de datos se desarrolló a través de la revisión bibliográfica de artículos originales, tanto nacionales como internacionales que tuvieron como tema principal: la gestión de residuos sólidos urbanos y su impacto medioambiental; primero se revisaron los títulos y resúmenes de las referencias encontradas y se seleccionaron los estudios más relevantes. Luego se revisó el texto completo de lo previamente seleccionado, con el fin de ratificar su elección. La técnica de análisis fue la revisión sistemática de los 10 artículos seleccionados, evaluándolos a cada uno para extraer la información más relevante, para su respectivo análisis. 


\section{RESULTADOS Y DISCUSIÓN}

\section{RESULTADOS}

\section{DATOS DE LA PUBLICACIÓN}

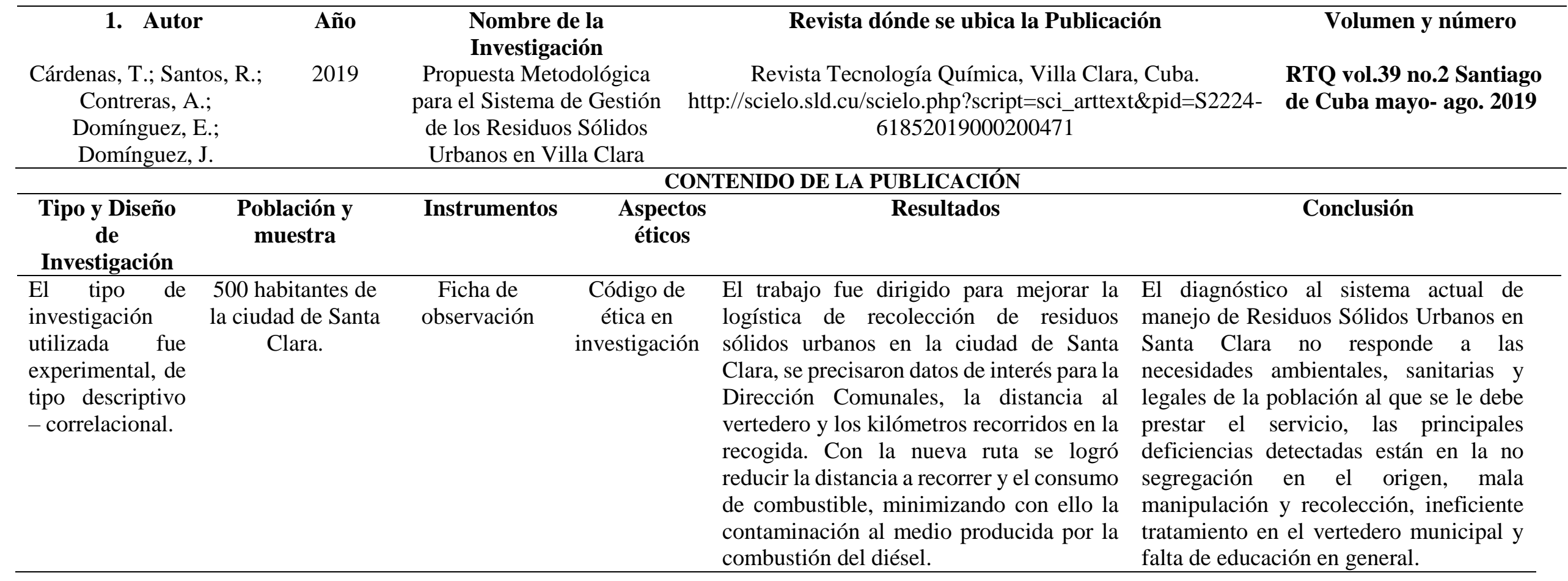




\begin{tabular}{|c|c|c|c|c|}
\hline 2. Autor & Año & Nombre de la Investigación & Revista dónde se ubica la Publicación & Volumen y número \\
\hline Rodríguez, J.; Ibarra, D. & 2019 & $\begin{array}{l}\text { Modelo para la evaluación } \\
\text { dinámica de la gestión de } \\
\text { residuos ordinarios de la } \\
\text { ciudad de Bogotá y su } \\
\text { influencia en el índice de } \\
\text { calidad ambiental urbana. }\end{array}$ & $\begin{array}{l}\text { Revista de Investigación Agraria y Ambiental, Bogotá, } \\
\text { Colombia. } \\
\text { file://C:/Users/J- } \\
\text { ModeloParaLaEvaluacionDinamicaDeLaGestionDeResiduo- } \\
\text { 6995812.pdf }\end{array}$ & Vol. 10, N. 2, 2019 \\
\hline
\end{tabular}

\begin{tabular}{|c|c|c|c|c|c|}
\hline $\begin{array}{l}\text { Tipo y Diseño de } \\
\text { Investigación }\end{array}$ & $\begin{array}{c}\text { Población y } \\
\text { muestra }\end{array}$ & Instrumentos & $\begin{array}{c}\text { Aspectos } \\
\text { éticos }\end{array}$ & Resultados & Conclusión \\
\hline $\begin{array}{ll}\text { El tipo } & \text { de } \\
\text { investigación } & \text { es } \\
\text { experimental, el } \\
\text { diseño } & \text { es } \\
\text { propositivo. } & \end{array}$ & $\begin{array}{l}04 \text { escenarios de } \\
\text { comportamiento }\end{array}$ & $\begin{array}{c}\text { Se utilizaron } \\
\text { simulaciones } \\
\text { del modelo }\end{array}$ & $\begin{array}{l}\text { Código de } \\
\text { ética en } \\
\text { investigación }\end{array}$ & $\begin{array}{l}\text { El primer escenario representa un caso en } \\
\text { el que se aumenta } 3 \text { veces la separación en } \\
\text { la fuente y las demás condiciones se } \\
\text { mantienen; el segundo escenario muestra } \\
\text { un } 20 \% \text { de todo tipo de aprovechamiento, } \\
\text { dividido en } 8,45 \% \text { para orgánicos y } 11,55 \\
\% \text { de reciclaje; al tercero, con las mismas } \\
\text { condiciones del segundo, se le suma un } \\
\text { aumento en } 3 \text { veces la separación en la } \\
\text { fuente; por último, el cuarto escenario se } \\
\text { caracteriza por un } 21,12 \% \text { de } \\
\text { aprovechamiento, } 28,88 \% \text { de reciclaje y } 3 \\
\text { veces la separación en la fuente. }\end{array}$ & $\begin{array}{l}\text { Se espera que esta investigación y sus } \\
\text { resultados sean útiles para la planeación de } \\
\text { la gestión de residuos sólidos en Bogotá, } \\
\text { así mismo se logrará que el manejo de } \\
\text { residuos sólidos no sea uno de los más } \\
\text { críticos, sino que, por lo contrario, se } \\
\text { convierta en la vía para mejorar la calidad } \\
\text { ambiental urbana, crear conciencia y } \\
\text { compromiso en la población e incrementar } \\
\text { la calidad de vida de los ciudadanos. }\end{array}$ \\
\hline
\end{tabular}

CONTENIDO DE LA PUBLICACIÓN

\begin{tabular}{|c|c|c|c|c|}
\hline 3. Autor & Año & Nombre de la Investigación & Revista dónde se ubica la Publicación & $\begin{array}{l}\text { Volumen y } \\
\text { número }\end{array}$ \\
\hline $\begin{array}{l}\text { Ruíz, D.; Suárez, J.; } \\
\text { Báez, J.; Saldívar, L. }\end{array}$ & 2017 & $\begin{array}{l}\text { Potencial de la transformación de residuos } \\
\text { sólidos urbanos en energía, a través del } \\
\text { Sistema de Oxidación por Batch en } \\
\text { Asunción, Paraguay }\end{array}$ & $\begin{array}{l}\text { Revista Población y Desarrollo, Asunción, Paraguay. } \\
\text { https://revistascientificas.una.py/index.php/RE/article/view/1280 }\end{array}$ & $\begin{array}{l}\text { N}^{\circ} .45 \text { (Población } \\
\text { y Desarrollo), } \\
\text { 2017, págs. 53-60 }\end{array}$ \\
\hline
\end{tabular}

Ciencia Latina Revista Científica Multidisciplinar, Ciudad de México, México.

ISSN 2707-2207 / ISSN 2707-2215 (en línea), julio-diciembre, 2020, Volumen 4, Número 2. https://doi.org/10.37811/cl_rcm.v4i2.135 p. 998. 


\begin{tabular}{|c|c|c|c|c|c|}
\hline $\begin{array}{l}\text { Tipo y Diseño de } \\
\text { Investigación }\end{array}$ & $\begin{array}{l}\text { Población y } \\
\text { muestra }\end{array}$ & Instrumentos & $\begin{array}{l}\text { Aspectos } \\
\text { éticos }\end{array}$ & Resultados & Conclusión \\
\hline $\begin{array}{l}\text { El tipo de } \\
\text { investigación fue } \\
\text { no experimental } \\
\text { de r enfoque } \\
\text { cualitativo, el } \\
\text { diseño rue } \\
\text { descriptivo. }\end{array}$ & 500 familias & $\begin{array}{l}\text { Recolección de } \\
\text { información y } \\
\text { observación, a } \\
\text { través de } \\
\text { revisión } \\
\text { documental y } \\
\text { fichas de } \\
\text { observación y } \\
\text { cálculo. }\end{array}$ & $\begin{array}{l}\text { Código de } \\
\text { ética en } \\
\text { investigación }\end{array}$ & $\begin{array}{l}\text { Utilizando el Sistema de Oxidación por Batch en } \\
\text { Asunción, se halló el cociente de } 28,91 / 0,055694 \text {, lo } \\
\text { cual nos da } 519 \text {, que es la cantidad de hogares que } \\
\text { podrían aprovechar diariamente la energía generada a } \\
\text { partir de los residuos sólidos de la ciudad. Si } \\
\text { consideramos la tarifa de la ANDE y traducimos esa } \\
\text { energía en dinero, es decir } 519 \text { hogares que paguen } \\
\text { mensualmente Gs. } 610.601 \text {, tenemos que los ingresos } \\
\text { brutos por mes en concepto de energía, utilizando este } \\
\text { sistema, serían de Gs. } 316.901 .919 \text {. }\end{array}$ & $\begin{array}{lr}\text { El método de oxidación de } \\
\text { Batch es una } \\
\text { eficienternativa } \\
\text { parcialmente } & \text { solucionar } \\
\text { problemática da la la } \\
\text { disposición de los desechos } \\
\text { y a su vez transformar los } \\
\text { desechos en energía limpia. }\end{array}$ \\
\hline
\end{tabular}

\section{CONTENIDO DE LA PUBLICACIÓN}

$\begin{array}{ccc}\text { 4. Autor } & \text { Año } & \text { Nombre de la Investigación } \\ \text { Mora, A.; Molina, N. } & 2017 & \begin{array}{c}\text { Diagnóstico del manejo de } \\ \text { residuos sólidos en el parque } \\ \text { histórico Guayaquil. }\end{array}\end{array}$

\section{Revista dónde se ubica la Publicación}

La granja, revista de ciencias de la vida, Ecuador. file:///C:/Users/J-

ARQU 1.UCV/AppData/Local/Temp/Dialnet-

DiagnosticoDelManejoDeResiduosSolidosEnElParqueHis-

$$
\text { 6094543.pdf }
$$

\begin{tabular}{|c|}
\hline $\begin{array}{l}\text { Tipo y Diseño de } \\
\text { Investigación }\end{array}$ \\
\hline $\begin{array}{ll}\text { El tipo } & \text { de } \\
\text { investigación } & \text { es } \\
\text { no experimental, } \\
\text { el diseño es } \\
\text { descriptivo. }\end{array}$ \\
\hline
\end{tabular}

\section{Población y} muestra

06 trabajadores muestra no probalística

Instrumentos

CONTENIDO DE LA PUBLICACIÓN

\section{Aspectos} éticos

La entrevista, y la observación de campo, para lo que se utilizó una hoja de registro
Consentimiento Sobre la transferencia y disposición final actual de informado.

los desechos especiales y peligrosos, no se evidencia una gestión acorde a la normativa. Los desechos especiales como aceite, luminarias y baterías se entregan al recolector municipal y tienen disposición final en el botadero municipal. Los desechos biopeligrosos no se han entregado hace dos años, excediendo el periodo de almacenamiento dispuesto por la autoridad.

\section{Volumen y número}

Vol. 26, No. 2, 2017, págs. 84-105

-




\begin{tabular}{|c|c|c|c|c|c|}
\hline 5. Autor & Año & \multicolumn{2}{|c|}{$\begin{array}{l}\text { Nombre de la } \\
\text { Investigación }\end{array}$} & Revista dónde se ubica la Publicación & Volumen y número \\
\hline Gamboa, V. & 2016 & $\begin{array}{l}\text { Gestión de res } \\
\text { urbanos en el } \\
\text { Chimbas, prox } \\
\text { Juan, Argentin } \\
\text { de la t }\end{array}$ & $\begin{array}{l}\text { siduos sólidos } \\
\text { departamento } \\
\text { vincia de San } \\
\text { na: la práctica } \\
\text { teoría. }\end{array}$ & $\begin{array}{c}\text { Revista Letras Verdes, Argentina. } \\
\text { https://revistas.flacsoandes.edu.ec/letrasverdes/article/view/1995 }\end{array}$ & $\begin{array}{c}\text { No. 20, } 2017 \text { (Ejemplar } \\
\text { dedicado a: Letras } \\
\text { Verdes } 20 \\
\text { (septiembre)), págs. 68- } \\
91\end{array}$ \\
\hline \multicolumn{6}{|c|}{ CONTENIDO DE LA PUBLICACIÓN } \\
\hline $\begin{array}{l}\text { Tipo y Diseño de } \\
\text { Investigación }\end{array}$ & $\begin{array}{l}\text { Población y } \\
\text { muestra }\end{array}$ & Instrumentos & $\begin{array}{l}\text { Aspectos } \\
\text { éticos }\end{array}$ & Resultados & onclusión \\
\hline $\begin{array}{l}\text { El tipo de } \\
\text { investigación fue } \\
\text { no experimental, } \\
\text { el diseño fue } \\
\text { descriptivo. }\end{array}$ & $\begin{array}{l}\text { La población } \\
\text { muestral se } \\
\text { trabajó con } \\
\text { datos primarios } \\
\text { y datos } \\
\text { secundarios. }\end{array}$ & $\begin{array}{l}\text { La entrevista, } \\
\text { y el análisis } \\
\text { documental. }\end{array}$ & $\begin{array}{l}\text { Código de } \\
\text { ética en } \\
\text { investigación }\end{array}$ & 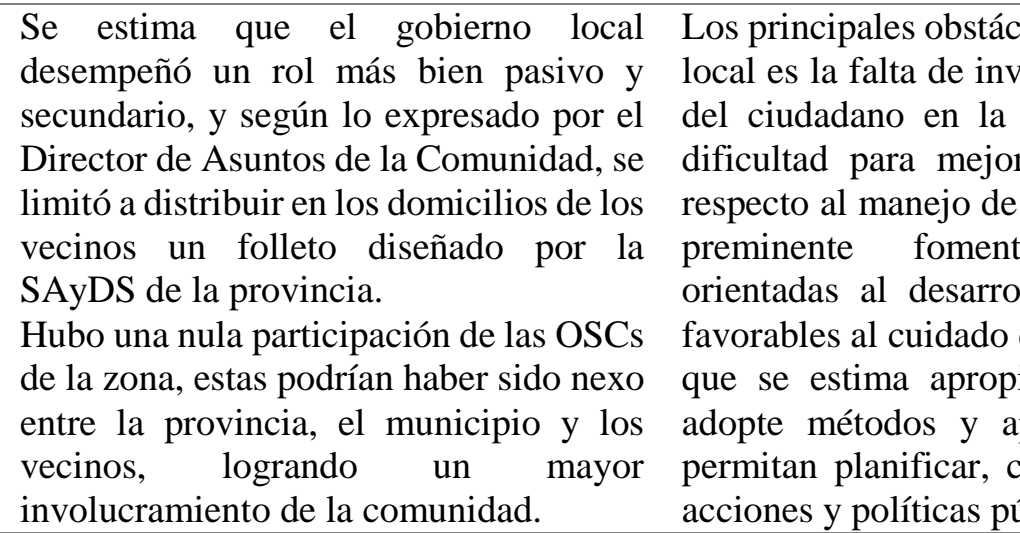 & $\begin{array}{l}\text { los que enfrenta el gobierno } \\
\text { lucramiento y colaboración } \\
\text { impieza de la ciudad y la } \\
\text { ar esos malos hábitos con } \\
\text { los RSU. Así mismo, que es } \\
\text { ir acciones educativas } \\
\text { lo de valores y conductas } \\
\text { lel medioambiente. Además, } \\
\text { ado que el ente municipal } \\
\text { lique instrumentos que le } \\
\text { ordinar, ejecutar y evaluar } \\
\text { blicas ambientales. }\end{array}$ \\
\hline
\end{tabular}

\begin{tabular}{ccccc}
\hline 6. Autor & Año & Nombre de la Investigación & Revista dónde se ubica la Publicación & Volumen y número \\
\hline Cabañas, E.; Díaz, M.; & 2019 & Densidad de los residuos & Revista de Investigación en Agro producción Sustentable, & Vol. 3, Núm. 1 \\
Oliva, M. & & sólidos de tres instituciones & Amazonas, Perú. \\
& & educativas de la ciudad de \\
Chachapoyas departamento de & http://revistas.untrm.edu.pe/index.php/INDESDOS/article/view/47 \\
& & Amazonas. & $9 / 593$
\end{tabular}

Ciencia Latina Revista Científica Multidisciplinar, Ciudad de México, México.

ISSN 2707-2207 / ISSN 2707-2215 (en línea), julio-diciembre, 2020, Volumen 4, Número 2. https://doi.org/10.37811/cl_rcm.v4i2.135 p. 1000. 


\section{CONTENIDO DE LA PUBLICACIÓN}

\begin{tabular}{|c|c|c|c|c|c|}
\hline $\begin{array}{c}\text { Tipo y Diseño } \\
\text { de } \\
\text { Investigación }\end{array}$ & $\begin{array}{c}\text { Población y } \\
\text { muestra }\end{array}$ & Instrumentos & Aspectos éticos & Resultados & Conclusión \\
\hline $\begin{array}{l}\text { El tipo de } \\
\text { estudio fue no } \\
\text { experimental, } \\
\text { con diseño } \\
\text { descriptivo - } \\
\text { correlacional. }\end{array}$ & $\begin{array}{c}\text { La muestra } \\
\text { tomada fue de los } \\
\text { docentes, } \\
\text { estudiantes y } \\
\text { personal } \\
\text { administrativo, de } \\
\text { las } 03 \\
\text { instituciones } \\
\text { educativas, y en } \\
\text { total sumaron } 494\end{array}$ & $\begin{array}{l}\text { La encuesta y } \\
\text { el instrumento } \\
\text { el cuestionario. }\end{array}$ & $\begin{array}{c}\text { Consentimiento } \\
\text { informado. }\end{array}$ & $\begin{array}{l}\text { El tipo de residuos sólidos que más se generan son } \\
\text { papeles, siendo el mayor porcentaje en la I.E Virgen } \\
\text { Asunta con un } 65,50 \% \text {, San Juan de la Libertad con un } \\
59,80 \% \text { y Seminario Jesús María con } 57,00 \% \text {. Por otro } \\
\text { lado, el promedio de humedad más alto se encontró en } \\
\text { San Juan de la Libertad con un } 82,20 \% \text {, seguido por } \\
\text { Virgen Asunta con } 78,72 \% \text { y Seminario Jesús María } \\
\text { con un } 75,06 \% \text {. Por otro lado la generación per cápita } \\
\text { (GPC) más alta, se encontró en la institución Seminario } \\
\text { Jesús María con un } 0,05 \% \text {, seguido con un } 0,04 \% \text { y un } \\
0,03 \% \text { para las instituciones de Virgen Asunta y San } \\
\text { Juan de la Libertad respectivamente. }\end{array}$ & $\begin{array}{l}\text { Tras la entrevista } \\
\text { determinó el tipo de } \\
\text { residuos sólidos que } \\
\text { consumen en las 03 I.E.; } \\
\text { estos datos permitieron } \\
\text { determinar cuáles son los } \\
\text { residuos sólidos para } \\
\text { elaborar planes de acción } \\
\text { certeros. Además, los tipos } \\
\text { de residuos que se generan } \\
\text { en cada institución pueden } \\
\text { ser reciclados o rehusados } \\
\text { en su gran mayoría. }\end{array}$ \\
\hline
\end{tabular}

\begin{tabular}{|c|c|c|c|c|}
\hline 7. Autor & Año & $\begin{array}{l}\text { Nombre de la } \\
\text { Investigación }\end{array}$ & Revista dónde se ubica la Publicación & Volumen y número \\
\hline $\begin{array}{l}\text { Salas, R.; Goñas, H.; } \\
\text { Sánchez, E. }\end{array}$ & 2018 & $\begin{array}{l}\text { Factores que influyen en el } \\
\text { manejo de los residuos } \\
\text { sólidos municipales, } \\
\text { Pomacochas, Amazonas. }\end{array}$ & $\begin{array}{l}\text { Revista de Investigación en Agro producción Sustentable, } \\
\text { Amazonas, Perú. } \\
\text { http://revistas.untrm.edu.pe/index.php/INDESDOS/article/view/382 }\end{array}$ & $\begin{array}{l}\text { Vol. 2, Núm. } 1 \\
\text { (2018) }\end{array}$ \\
\hline
\end{tabular}

\begin{tabular}{|c|c|c|c|c|c|}
\hline \multicolumn{6}{|c|}{ CONTENIDO DE LA PUBLICACIÓN } \\
\hline $\begin{array}{l}\text { Tipo y Diseño } \\
\text { de } \\
\text { Investigación }\end{array}$ & $\begin{array}{c}\text { Población y } \\
\text { muestra }\end{array}$ & Instrumentos & Aspectos éticos & Resultados & Conclusión \\
\hline $\begin{array}{l}\text { El tipo de } \\
\text { estudio fue no } \\
\text { experimental, }\end{array}$ & $\begin{array}{l}\text { La muestra } \\
\text { tomada fue de } \\
136 \text { viviendas, }\end{array}$ & $\begin{array}{l}\text { La encuesta y } \\
\text { el instrumento } \\
\text { el cuestionario. }\end{array}$ & $\begin{array}{c}\text { Consentimiento } \\
\text { informado. }\end{array}$ & $\begin{array}{l}\text { Los resultados en cuanto a la } \\
\text { influencia de los factores sociales y } \\
\text { ambientales, evidenciaron que para }\end{array}$ & $\begin{array}{l}\text { Para el manejo de los residuos sólidos } \\
\text { municipales, los factores más influyentes son el } \\
\text { social y el político, puesto que existe }\end{array}$ \\
\hline
\end{tabular}

Ciencia Latina Revista Científica Multidisciplinar, Ciudad de México, México.

ISSN 2707-2207 / ISSN 2707-2215 (en línea), julio-diciembre, 2020, Volumen 4, Número 2. https://doi.org/10.37811/cl rcm.v4i2.135 p. 1001 


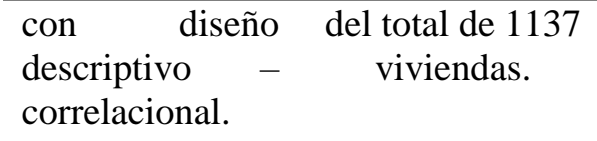

correlacional. realizar un manejo eficiente de los residuos sólidos municipales, es necesario involucrar a la población y educar en temas ambientales para que se sientan parte de esta problemática y contribuyan al cuidado del medio ambiente. desconocimiento por parte de la población en el manejo adecuado de los residuos sólidos municipales y el desinterés de la autoridad local frente a esta problemática; la disposición final es un dilema, la aparición de botaderos informales incrementan la contaminación ambiental, afectando la salud de la población.

\begin{tabular}{|c|c|c|c|c|}
\hline 8. Autor & Año & Nombre de la Investigación & Revista dónde se ubica la Publicación & Volumen y número \\
\hline $\begin{array}{l}\text { Sánchez, M.; Cruz, J.; } \\
\text { Giraldo, J. }\end{array}$ & 2019 & $\begin{array}{l}\text { Análisis de la opinión de los } \\
\text { hogares sobre la gestión de los } \\
\text { residuos sólidos domiciliarios en } \\
\text { Bogotá }\end{array}$ & $\begin{array}{c}\text { Revista Semestre económico. } \\
\text { https://doi.org/10.22395/seec.v22n52a5 }\end{array}$ & $\begin{array}{l}\text { Vol. } 22 \text { Issue 52, p97-129. } \\
\text { 33p. }\end{array}$ \\
\hline
\end{tabular}

\begin{tabular}{|c|c|c|c|c|c|}
\hline \multicolumn{6}{|c|}{ CONTENIDO DE LA PUBLICACIÓN } \\
\hline $\begin{array}{l}\text { Tipo y Diseño de } \\
\text { Investigación }\end{array}$ & $\begin{array}{c}\text { Población y } \\
\text { muestra }\end{array}$ & Instrumentos & $\begin{array}{l}\text { Aspectos } \\
\text { éticos }\end{array}$ & Resultados & Conclusión \\
\hline $\begin{array}{l}\text { El tipo de estudio } \\
\text { fue no } \\
\text { experimental, se } \\
\text { tomó como diseño } \\
\text { el descriptivo. }\end{array}$ & $\begin{array}{l}384 \text { hogares, } \\
\text { método de } \\
\text { muestreo } \\
\text { estratificado. }\end{array}$ & Encuesta & $\begin{array}{c}\text { Consentimiento } \\
\text { informado. }\end{array}$ & $\begin{array}{l}\text { De los } 384 \text { encuestados, el } 77 \% \text { eran } \\
\text { conscientes de la importancia de proteger } \\
\text { el medio ambiente para mejorar la calidad } \\
\text { de vida, lo que los motiva a preocuparse } \\
\text { por el adecuado manejo de los residuos } \\
\text { sólidos. Además, se encontró que el } \\
17,2 \% \text { de los encuestados se sentía poco } \\
\text { o nada afectado por la inadecuada } \\
\text { disposición de los desechos, mientras que } \\
101 \text { personas }(26,3 \%) \text { respondieron } \\
\text { sentirse muy afectados. Asimismo, } 164 \\
\text { encuestados }(42,7 \%) \text { se sintieron }\end{array}$ & $\begin{array}{l}\text { Es preciso que la educación ambiental } \\
\text { trascienda hacia la cultura ambiental, } \\
\text { para que las acciones de los actores } \\
\text { redunden en una gestión de residuos } \\
\text { sólidos coherente con la economía } \\
\text { circular. Por ello, se requieren } \\
\text { campañas educativas precisas, cortas y } \\
\text { contundentes. Además, los planes de } \\
\text { gestión integral de residuos sólidos } \\
\text { deben ser vinculantes entre el Estado, la } \\
\text { empresa privada y los ciudadanos. Debe } \\
\text { ser el Estado quien realice el control y } \\
\text { garantice el cumplimiento de la norma. }\end{array}$ \\
\hline
\end{tabular}




\begin{tabular}{ccccc}
\hline 9. Autor & Año & Nombre de la Investigación & Revista dónde se ubica la Publicación & Volumen y número \\
& 2016 & $\begin{array}{c}\text { Actitudes y prácticas ambientales } \\
\text { de la población de la ciudad de } \\
\text { Tumi, J. }\end{array}$ & $\begin{array}{c}\text { Espacio Abierto Cuaderno Venezolano de } \\
\text { Puno, Perú sobre gestión de } \\
\end{array}$ & Sol.25 No.4 (octubre - \\
& residuos sólidos. & diciembre, 2016) \\
& & &
\end{tabular}

\begin{tabular}{|c|c|c|c|c|c|}
\hline $\begin{array}{l}\text { Tipo y Diseño de } \\
\text { Investigación }\end{array}$ & $\begin{array}{l}\text { Población y } \\
\text { muestra }\end{array}$ & Instrumentos & $\begin{array}{l}\text { Aspectos } \\
\text { éticos }\end{array}$ & Resultados & Conclusión \\
\hline $\begin{array}{l}\text { El tipo de estudio } \\
\text { fue no } \\
\text { experimental, } \\
\text { transversal, } \\
\text { explicativo } \\
\text { correlacional. }\end{array}$ & $\begin{array}{c}\text { La población } \\
\text { muestral lo } \\
\text { conformaron } 384 \\
\text { personas. }\end{array}$ & $\begin{array}{c}\text { Encuesta, } \\
\text { aplicada a través } \\
\text { de un } \\
\text { cuestionario, } \\
\text { complementado } \\
\text { con observación } \\
\text { participante y } \\
\text { entrevistas a } \\
\text { profundidad a } \\
\text { actores sociales } \\
\text { clave. }\end{array}$ & $\begin{array}{l}\text { Consentimiento } \\
\text { informado. }\end{array}$ & $\begin{array}{l}\text { Por consiguiente, como Fc } \\
\text { cae en la región de rechazo, } \\
\text { se rechaza la hipótesis Ho y } \\
\text { se acepta H1: Existe } \\
\text { influencia del acceso a } \\
\text { servicios básicos, el } \\
\text { almacenamiento y } \\
\text { disposición final de RS y la } \\
\text { localización espacial de la } \\
\text { vivienda respecto a la bahía } \\
\text { del Lago Titicaca sobre las } \\
\text { actitudes en la gestión de } \\
\text { residuos sólidos. }\end{array}$ & $\begin{array}{l}\text { Las prácticas de sanidad e higiene son adecuadas en } \\
\text { la mayoría de familias de la ciudad de Puno, } \\
\text { expresada en el } 77 \% \text { de familias que realizan la } \\
\text { evacuación de heces en el baño de domicilio o } \\
\text { servicios públicos, el } 60 \% \text { realizan siempre el } \\
\text { lavado de las manos después de manipular los RS, } \\
\text { el } 56 \% \text { realizan la limpieza del baño en forma diaria } \\
\text { o interdiaria y el } 61 \% \text { de familias hierve el agua de } \\
\text { beber. Esta situación es expresión de la mejora de la } \\
\text { conciencia sanitaria de la población y la limitada } \\
\text { cobertura del programa de educación ambiental; } \\
\text { agudizada por el colapso de la laguna de oxidación } \\
\text { ubicada en la bahía del Lago Titicaca. }\end{array}$ \\
\hline
\end{tabular}

\begin{tabular}{ccccc} 
10. Autor & Año & Nombre de la Investigación & Revista dónde se ubica la Publicación & Volumen y número \\
$\begin{array}{c}\text { Ferreira, R.; Borga, T.; } \\
\text { Sartorel, A. }\end{array}$ & 2017 & $\begin{array}{c}\text { Diagnóstico de residuos sólidos } \\
\text { urbanos de municipio, mediante un } \\
\text { análisis cuantitativo y cualitativo }\end{array}$ & Revista Geogr. Académica, Brasil. & v.11, n.1 (viii.2017) \\
& & & \\
\hline
\end{tabular}




\section{CONTENIDO DE LA PUBLICACIÓN}

\begin{tabular}{|c|c|c|c|c|c|}
\hline $\begin{array}{l}\text { Tipo y Diseño } \\
\text { de } \\
\text { Investigación }\end{array}$ & $\begin{array}{c}\text { Población y } \\
\text { muestra }\end{array}$ & Instrumentos & $\begin{array}{l}\text { Aspectos } \\
\text { éticos }\end{array}$ & Resultados & Conclusión \\
\hline $\begin{array}{l}\text { Técnica de } \\
\text { caracterización } \\
\text { gravimétrica con } \\
\text { el análisis }\end{array}$ & $\begin{array}{l}\text { La población } \\
\text { muestral lo } \\
\text { conformaron } 45 \\
\text { residencias }(128\end{array}$ & $\begin{array}{l}\text { Método } \\
\text { estadístico de } \\
\text { muestreo } \\
\text { sistemático es }\end{array}$ & $\begin{array}{l}\text { Consentimiento } \\
\text { informado. }\end{array}$ & $\begin{array}{l}\text { Según Fuzaro y Ribeiro (2007), el sistema de } \\
\text { recolección selectiva es un método eficiente para } \\
\text { reducir los impactos ambientales, sin embargo, para } \\
\text { obtener una buena gestión y calidad ambiental en el }\end{array}$ & $\begin{array}{l}\text { Por lo tanto, el presente } \\
\text { estudio tiene como objetivo } \\
\text { ayudar a la guía de residuos } \\
\text { sólidos urbanos, y el }\end{array}$ \\
\hline $\begin{array}{l}\text { cualitativo y } \\
\text { cuantitativo de } \\
\text { los residuos } \\
\text { sólidos } \\
\text { municipales. }\end{array}$ & habitantes) & $\begin{array}{l}\text { al menos el } \\
10 \% \text { de la } \\
\text { muestra total. }\end{array}$ & & $\begin{array}{l}\text { proceso, se debe insertar a toda la población y participar } \\
\text { en el proceso de separación de residuos sólidos. El } \\
\text { monitoreo de la generación de residuos sólidos con la } \\
\text { caracterización gravimétrica de los residuos permite } \\
\text { diagnosticar y mejorar el desempeño de la educación } \\
\text { ambiental vinculada al programa de recolección } \\
\text { selectiva. (REZENDE et al., 2013; CAMPOS; BORGA, } \\
\text { 2015) }\end{array}$ & $\begin{array}{l}\text { municipio del estudio no } \\
\text { tiene el Plan Municipal para } \\
\text { la Gestión Integrada de } \\
\text { Residuos Sólidos Urbanos - } \\
\text { PMIGRSU, ni presenta } \\
\text { ningún análisis cuantitativo y } \\
\text { cualitativo de los residuos } \\
\text { sólidos urbanos. }\end{array}$ \\
\hline
\end{tabular}




\section{DISCUSIÓN}

Posterior a la revisión sistemática de los artículos, encontramos que del 100\% corresponde a América latina y el Caribe, resaltando los países de Colombia y Perú con el $20 \%$ de publicaciones respectivamente, seguido de los países de Cuba, Paraguay, Ecuador, Argentina, Venezuela y Brasil que alcanzaron 10\% cada uno. En relación a los diseños y tipos de estudios el 100\% son descriptivos con sus diferentes variantes.

En relación a la gestión de residuos sólidos urbanos y su impacto medioambiental podemos resaltar que Cárdenas, Santos, Contreras, Domínguez, E.; Domínguez, J., establecen que, el diagnóstico del sistema de manejo de residuos sólidos urbanos no responde a las necesidades ambientales, sanitarias y legales. Por ello debe desarrollarse un plan estratégico ambiental que garantice el beneficio a los ciudadanos. También Rodríguez, J.; Ibarra, D., determina que la investigación contribuye al manejo de residuos sólidos para vencer el crítico actual del sistema. Por su lado Ruíz, D.; Suárez, J.; Báez, J.; Saldívar, L, concluye que, el método de Batch es una alternativa para garantizar la disposición de los desechos y transformarlos en energía limpia.

Por su parte, Mora, A.; Molina, N. establece en su estudio que, la transferencia y disposición final actual de los desechos especiales y peligrosos no evidencian una gestión acorde a la normativa, por lo que es necesario que la normativa sea socializada y determinante para que el equipo local pueda gestionar la seguridad y eliminación de residuos sólidos. Por su parte Gamboa, V., estableció que, que el gobierno local desempeñó un rol pasivo y secundario, limitándose sólo a distribuir en los domicilios de los vecinos folletos que no fortalece la intervención. También Cabañas, E.; Díaz, M.; Oliva, M., indicaron que, el tipo de residuos sólidos que más se generan son papeles, en las diferentes instituciones educativas, por ende, puede penarse en desarrollar una condición de reciclaje de estos residuos.

Sin embargo, Salas, R.; Goñas, H.; Sánchez, E, establecen que, la influencia de los factores sociales y ambientales, evidenciaron que para realizar un manejo eficiente de los residuos sólidos municipales, es necesario involucrar a la población y educar en temas ambientales. Asimismo, Sánchez, M.; Cruz, J.; Giraldo, J., determinó que, es preciso que la educación ambiental trascienda hacia la cultura ambiental, para que las acciones de los actores redunden en una gestión de residuos sólidos coherente con la economía circular. Igualmente, Tumi, J, que las prácticas de sanidad e higiene son adecuadas en la mayoría de 
familias por ende se garantiza una adecuada eliminación de residuos sólidos y en especial de protección del ambiente.

Además, Ferreira, R.; Borga, T.; Sartorel, A., determinaron que, el sistema de recolección selectiva es método eficiente para reducir los impactos ambientales, sin embargo, para obtener una buena gestión y calidad ambiental en el proceso, se debe insertar a toda la población y participar en el proceso de separación de residuos sólidos, ello debe generar responsabilidad en la población asegurando el beneficio social.

\section{CONCLUSIÓN O CONSIDERACIONES FINALES}

El manejo de los residuos sólidos urbanos por parte de las municipalidades se identificaron como factores más influyentes la condición social y política, puesto que la población desconoce el manejo adecuado de los residuos sólidos, y muchas veces existe el desinterés de la autoridad local frente a esta problemática, afectando significativamente al mejoramiento del proceso integral que afrontan entre otros aspectos: la disposición final es un dilema, la aparición de botaderos informales incrementan la contaminación ambiental, afectando la salud de la población.

La mejora de la conciencia sanitaria de la población, así mismo juega en contra, la limitada cobertura del programa de educación ambiental; la coyuntura actual que estamos viviendo con la propagación de la pandemia del covid 19, podría darnos luces en cuanto a un acercamiento a la población con planes de capacitación de modo remoto, lo que

significaría un gran avance en el mejoramiento de esta problemática que nos afecta hoy en día. Asimismo, esta situación es una oportunidad para que se promuevan negocios del reciclaje en sus distintos ámbitos, como por ejemplo el plástico para ser reutilizado como material de construcción, o los desechos digitales que podrían ser reutilizados en bisutería y otros.

La educación ambiental debería trascender hacia la cultura ambiental, para que las acciones de los actores redunden en una gestión de residuos sólidos coherente con la economía circular. Por ello, se requieren campañas educativas precisas, cortas y contundentes. Además, los planes de gestión integral de residuos sólidos deben ser vinculantes entre el Estado, la empresa privada y los ciudadanos. Debe ser el Estado quien realice el control y garantice el cumplimiento de la norma. 


\section{LISTA DE REFERENCIAS}

Cabañas, E.; Díaz, M.; Oliva, M. (2019). Densidad de los residuos sólidos de tres instituciones educativas de la ciudad de Chachapoyas departamento de Amazonas. Revista de Investigación en Agro producción Sustentable, Amazonas, Perú, 3. http://revistas.untrm.edu.pe/index.php/INDESDOS/article/view/479/593

Cárdenas, T.; Santos, R.; Contreras, A.; Domínguez, E.; Domínguez, J. (2019). Propuesta Metodológica Para el Sistema de Gestión de los Residuos Sólidos Urbanos en Villa Clara. Tecnología Química, 39(2), 464-483.

Ferreira, R.; Borga, T.; Sartorel, A. (2017). Diagnóstico de residuos sólidos urbanos de municipio, mediante un análisis cuantitativo y cualitativo. Letras Verdes, 20, 68-90. https://doi.org/10.17141/letrasverdes.20.2017.1995

Gamboa, V., \& Lahoz, E. (2016). Gestión de residuos sólidos urbanos en el departamento Chimbas, provincia de San Juan, Argentina: la práctica de la teoría. Letras $\quad$ Verdes, $20, \quad 68-90$. https://doi.org/10.17141/letrasverdes.20.2017.1995.

Mora, A.; Molina, N. (2017). Diagnóstico del manejo de residuos sólidos en el parque histórico Guayaquil. La granja, revista de ciencias de la vida, Ecuador, 26, 84 -105 .

Rodríguez, J. \& Ibarra, D. (2019). Modelo para la evaluación dinámica de la gestión de residuos ordinarios de la ciudad de Bogotá y su influencia en el índice de calidad ambiental urbana. Revista de Investigación Agraria y Ambiental, Bogotá, Colombia.

Ruíz, D.; Suárez, J.; Báez, J.; Saldívar, L. (2017). Potencial de la transformación de residuos sólidos urbanos en energía, a través del Sistema de Oxidación por Batch en Asunción, Paraguay. Población y Desarrollo, 45, 53 - 60. https://revistascientificas.una.py/index.php/RE/article/view/1280

Salas, R.; Goñas, H.; Sanchez, E. (2018). Factores que influyen en el manejo de los residuos sólidos municipales, Pomacochas, Amazonas. Agro producción Sustentable, http://revistas.untrm.edu.pe/index.php/INDESDOS/article/view/382 
Sánchez, M.; Cruz, J.; Giraldo, J. (2019). Análisis de la opinión de los hogares sobre la gestión de los residuos sólidos domiciliarios en Bogotá. Semestre Económico, 22(52), 97-129. https://doi.org/10.22395/seec.v22n52a5

Tumi, J. (2016). Actitudes y prácticas ambientales de la población de la ciudad de Puno, Perú sobre gestión de residuos sólidos. Revista Geográfica Académica, $11,1$. 